Case report

\title{
Gliomatosis cerebri type II: two case reports
}

Pietro Ivo D'Urso ${ }^{1}$, Oscar Fernando D'Urso ${ }^{2 *}$, Santo Marsigliante ${ }^{2}$, Carlo Storelli $^{2}$, Alessandro Distante ${ }^{3}$, Francesca Sanguedolce ${ }^{4}$, Antonia Cimmino ${ }^{4}$, Giuseppe Luzi ${ }^{5}$, Cosimo Damiano Gianfreda ${ }^{6}$, Antonio Montinaro ${ }^{6}$ and Pasqualino Ciappetta ${ }^{1}$

Addresses: ${ }^{1}$ Department of Neurosciences - Section of Neurosurgery, University of Bari Medical School, Bari, Italy, ${ }^{2}$ Department of Biological and Environmental Sciences and Technologies (DiSTeBA), Ecotekne, Via Prov.le per Monteroni, 73100 Lecce, Italy, ${ }^{3}$ CNR-IFC Lecce section, Ecotekne, Via Prov.le per Monteroni, 73100 Lecce, Italy, ${ }^{4}$ Department of Pathology - Section of Neuropathology, University of Bari Medical School, Bari, Italy, ${ }^{5}$ Department of Clinical Immunology and Allergology, Sant'Anrea Hospital, Rome, Italy and ${ }^{6}$ Neurosurgery Operative Unit, V. Fazzi Hospital, 73100 Lecce, Italy

Email: PIDU - pietrivo@libero.it; OFDU* - durso@biotecgen.it; SM - santo.marsigliante@unile.it; CS - carlo.storelli@unile.it; AD - distante@isbem.it; FS - sangeudolce@libero.it; AC - acimmino@libero.it; GL - luziglobal@hotmail.com; CDG - 3383383244@tim.it; AM - a.montinaro@lbero.it

* Corresponding author

Received: 7 July 2008 Accepted: 23 January 2009 Published: 15 June 2009

Journal of Medical Case Reports 2009, 3:7225 doi: 10.4076/1752-1947-3-7225

This article is available from: http://jmedicalcasereports.com/jmedicalcasereports/article/view/7225

(C) 2009 D'Urso et al; licensee Cases Network Ltd.

This is an Open Access article distributed under the terms of the Creative Commons Attribution License (http://creativecommons.org/licenses/by/3.0), which permits unrestricted use, distribution, and reproduction in any medium, provided the original work is properly cited.

\begin{abstract}
Introduction: Two types of gliomatosis cerebri exist: Type I and Type II. We report the results of a histological and genetic study of two cases of gliomatosis cerebri Type II, correlating these results with therapy and prognosis.

Case presentation: Two patients, a 52-year-old man (Patient I) and a 76-year-old man (Patient 2) with gliomatosis cerebri II were admitted to our institution; they underwent surgical treatment and received radiotherapy and chemotherapy. At the 24-month follow-up, Patient I was still alive, while Patient 2 had died. The poor prognosis of Patient 2 was underlined by molecular analysis which showed that the angiogenesis related genes VCAMI and VEGF were overexpressed, reflecting the high degree of neovascularization.

Conclusion: Genes involved in drug resistance and metallothioneins were highly expressed in Patient 2 and this, associated with unmethylated O6-methylguanine methyltransferase, can explain the lack of response to chemotherapy.
\end{abstract}

\section{Introduction}

Gliomatosis cerebri (GC) is a diffuse, frequently bilateral, glial tumor which infiltrates the brain, involving more than two lobes. It often extends to the infratentorial structures and even to the spinal cord. According to the current WHO classification of brain tumors, GC is a distinct malignant neuroepithelial neoplasm of uncertain origin [1]. Pathologists describe two types of GC: Type I is the classic form of GC characterized by diffuse overgrowth with neoplastic glial elements without a focal mass 
presence. Type II may stem from Type I and is characterized by a diffuse brain infiltration and focal mass presence, usually a high-grade glioma [1]. More than 200 cases of GC have been described in the literature, but very few cases of Type II GC have been genetically typed. We report the results of a histological and genetic study in two cases of gliomatosis cerebri Type II. A prognostic correlation of these results is also provided.

\section{Case presentation}

Two patients of Italian ethnicity, a 52-year-old man (Patient 1) and a 76-year-old man (Patient 2), with clinical signs of increased intracranial pressure were admitted to our institution. Pre-operative neuroradiological studies (computed tomography (CT) scan and magnetic resonance imaging (MRI)) were performed. The patients underwent surgical treatment and received radiotherapy at a 54.9 Gy dose and concomitant chemotherapy with temozolomide.

Fresh surgical specimens were frozen in liquid nitrogen immediately after removal, and were treated immediately upon arrival at the laboratory. Isolation of DNA from these specimens was performed using the ChargeSwitch ${ }^{\circledR}$ gDNA Micro Tissue Kit (Invitrogen, Karlsruhe, Germany). Isolation of total RNA from the surgical specimens was performed using the PureLink ${ }^{\mathrm{TM}}$ Micro-toMidi ${ }^{\mathrm{TM}}$ Total RNA Purification System following the manufacturer's instructions (Invitrogen).

TP53 exons 5-8 and all PTEN exons were analyzed by the sequencing of various tumor regions. Each exon was polymerase chain reaction (PCR) amplified. Sequence reactions were performed with a BigDye ${ }^{\circledR}$ Terminator v3.1kit (Applied Biosystems, Warrington, UK) using both forward and reverse primers. Finally, the sequences were read by an automated sequence reader (ABI PRISM 3130, Applied Biosystems).

For CGH analysis, a custom 60-mer amino modified oligo-microarray was used, containing 6000 spots, specific to 287 chromosomal regions implicated in cancer. Labeling reactions were performed on purified restricted DNA using the BioPrime Array CGH Genomic Labeling System (Invitrogen). Experimental and reference targets were hybridized for 14 to 18 hours at $42^{\circ} \mathrm{C}$. After washing, slides were scanned using an Affymetrix 428 array scanner (Affymetrix, Santa Clara, CA, USA).

For LOH analysis, a custom 30-mer amino modified oligomicro array was used. Single nucleotide polymorphisms (SNPs) were chosen to represent markers for 2q14.3, 2q22.1, 3q13, 7q22, 10q22-10q23, 12q14, 13q14, 16q22 17p.13.1, and 19q13.2. Two 30-mer oligonucleotides per locus were designed to represent the alternate alleles of each SNP. Each oligonucleotide consisted of a 12-nucleotide hairpin and an 18- to 21-nucleotide target sequence with the polymorphic base in the middle.

A custom 60-mer amino modified oligo-array was used, containing 800 probes specific for genes commonly altered in cancer. RNA was labelled using the SuperScript Indirect RNA Amplification System (Invitrogen). The resulting amplified amino-allyl aRNA was dyed using Cy3 for test samples and Cy5 for reference samples.

The microarray expression results were validated by quantitative real-time PCR, using an ABI 7500 Real-Time PCR System (Applied Biosystems). The Wilcoxon signedrank test was used to analyze statistical significance.

The DNA methylation patterns in the CpG island of the O6-methylguanine-DNA methyltransferase (MGMT), p16, deleted in colorectal cancer (DCC) and death-associated protein kinase 1 (DAPK1) genes were determined by sodium bisulfite treatment and subsequent methylationspecific PCR MS PCR. Primers for MS PCR were designed to encompass the CpG-rich area of the promoter region around the transcription initiation site.

The neuroradiological studies (CT scan and MRI) indicated gliomatosis cerebri Type II. Both patients underwent surgical removal of the focal mass and biopsies in the adjacent areas, and received temozolomide and radiation therapy postoperatively. At the 24-month follow-up, after chemo- and radiation therapy, Patient 1 was still alive with impaired neurological conditions, while Patient 2 had died.

The histological study on the removed masses revealed a diffuse proliferation of neoplastic spindle cells with rodshaped nuclei infiltrating the brain parenchyma in both patients. Such cells appeared arranged in short chains parallel to nervous fibers, in the shape of a high-grade malignant neoplasm. These aspects were compatible with the pre-operative diagnosis of gliomatosis cerebri Type II. Paraffin-embedded sections were stained by the immunoperoxidase method which yielded the following results: positive staining reaction to glial fibrillary acidic protein (GFAP) and to S100 protein (Figure 1).

In Patient 1, bidirectional sequencing revealed a mutation in codon 248 of TP53, with arginine to tryptophan substitution (Figure 2a). Patient 2 carried an intronic point mutation in the PTEN gene, which altered the splice donor site within intron 2 and therefore likely caused expression of an aberrantly spliced mRNA (Figure $2 \mathrm{~b}$ ). These same mutations were observed in all of the tumor regions.

CGH analysis did not detect any CDKN2A (9p21) deletion (confirmed by $\mathrm{LOH}$ ) and no CDK4 or MDM2 amplification in either patient. Patient 2 showed a gain in the EGFR 

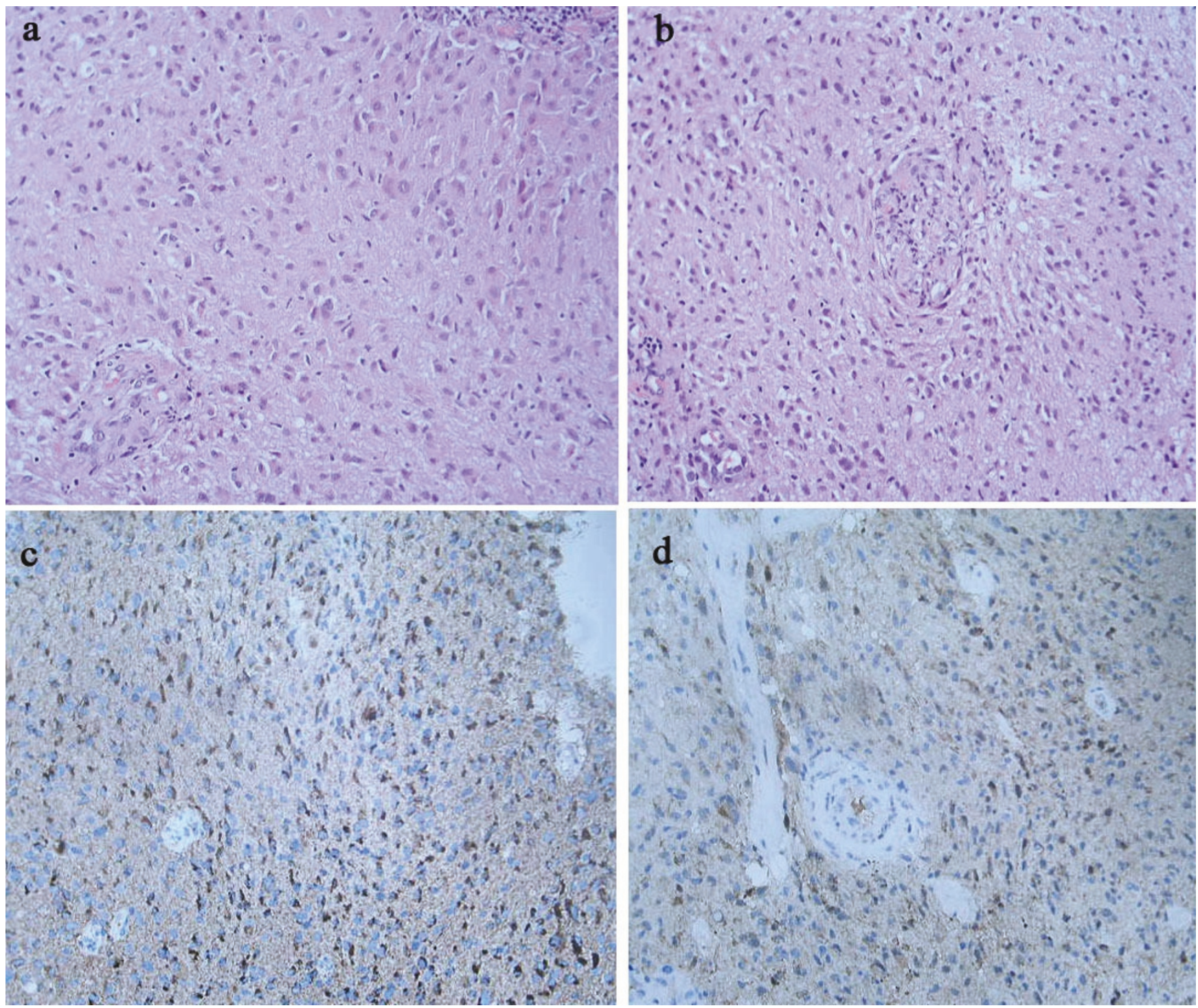

Figure I. Histological study of the removed masses revealed a diffuse proliferation of neoplastic spindle cells with rod-shaped nuclei infiltrating the brain parenchyma in both Patients I (a) and 2 (b). Paraffin-embedded sections were stained by the immunoperoxidase method and were positive for glial fibrillary acidic protein (GFAP) (c) and SI00 protein (d).

gene copy number. These analyses revealed the deletion of the p27kip1 gene locus, no aberrations of CDKN1A (p21), and losses of $2 \mathrm{q}, 3 \mathrm{p}, 16 \mathrm{q}$ and $19 \mathrm{q}$ and gains of $7 \mathrm{q}$ in both patients (data not shown).

The results of cDNA gene expression profiling are reported in Table 1, while Figure 3a shows a scatterplot with a 2 -fold threshold. Genes found to be overexpressed were:

- transcriptional regulators (including ZNF258, EYA2, EGR1, JUNB) and intracellular signal transduction genes (RGS7, EHD3, CS1, ITPK1, GRB2, STK2) in both tumors;
- angiogenesis related genes (VCAM1, VEGF) and drug resistance genes (ABCC3, MTIL) in Patient 2.

Genes found to be down-regulated were:

- signal transduction genes (PSHL, P1M1, IQGAP, RDC1, RGS16) and cytoskeleton related genes (PFN1, MSN, PLEK, VIM, CAPG) in Patient 2;

- apoptosis genes (CASP7, BCLW, DAP3 and DCC in line with methylation status) in both tumors. 

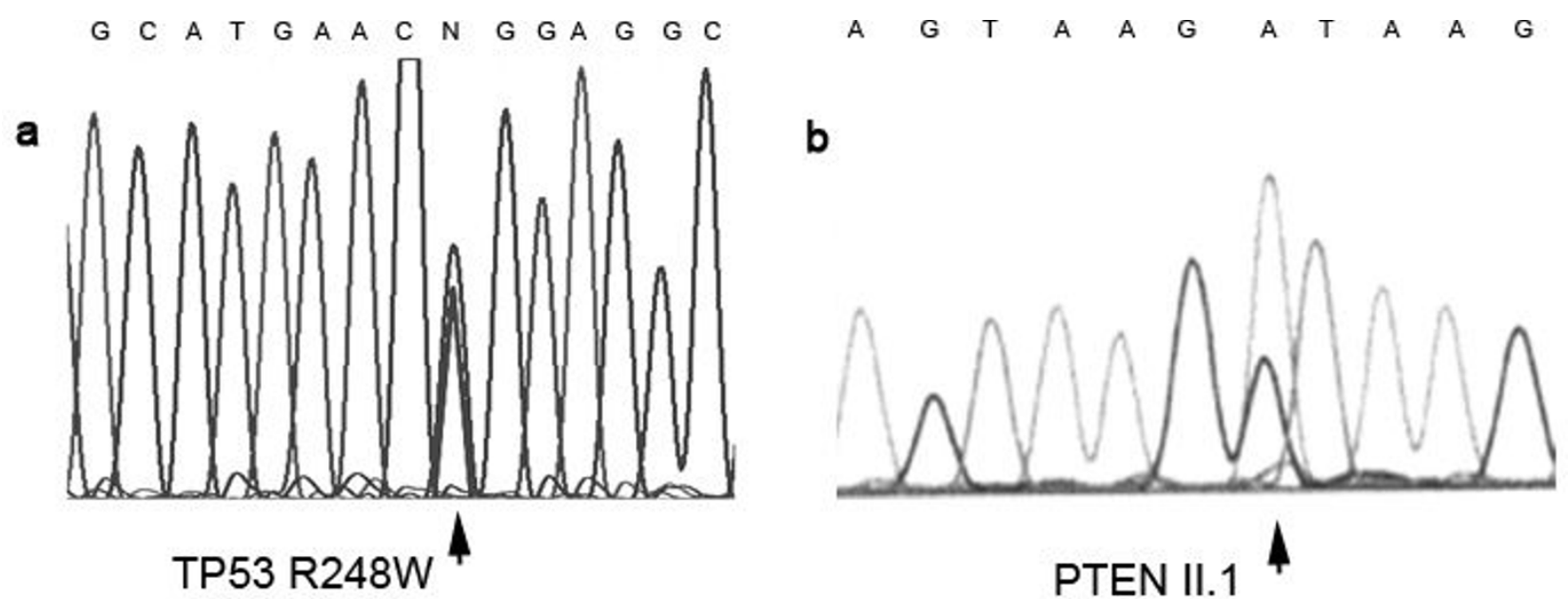

Figure 2. (a) Sequence around codon 248 of TP53 showing the mutated base which determines an arginine to tryptophan substitution. (b) The PTEN point mutation at the intron 2 splice donor site causes a defective splicing which likely results in a non-functional protein.

Table I. Microarray normalized data

\begin{tabular}{|c|c|c|c|}
\hline Name & Description & I/C* Patient I & I/C* Patient 2 \\
\hline \multicolumn{4}{|c|}{ Under-expressed in Patient 2} \\
\hline IQGAP & IQ motif containing GTPase-activating protein I & 1.13 & 0.02 \\
\hline ITPKI & Inositol I,3,4-triphosphate 5/6 kinase & 1.5 & 1.6 \\
\hline PIMI & Pim-I proto-oncogene gene & 0.99 & 0.06 \\
\hline PSHL & Phosphoserine phosphatase-like & 1.0 & 0.12 \\
\hline PTGER4 & Prostaglandin E receptor 4 (subtype EP4) & 0.18 & 0.16 \\
\hline $\mathrm{RDCl}$ & G protein-coupled receptor & 1.0 & 0.11 \\
\hline RGSI6 & Regulator of G-protein signaling 16 & 1.12 & 0.06 \\
\hline CAPG & Capping protein (actin filament), gelsolin-like & 1.1 & 0.2 \\
\hline MSN & Moesin & 1.0 & 0.1 \\
\hline PFNI & Profilin I & 0.98 & 0.12 \\
\hline PLEK & Plekstrin & 1.12 & 0.11 \\
\hline VIM & Vimentin & 1.16 & 0.03 \\
\hline \multicolumn{4}{|c|}{ Over-expressed in Patient 2} \\
\hline VCAMI & Vascular cell adhesion molecule I & 1.01 & 4.8 \\
\hline VEGF & Vascular endothelial growth factor & 1.0 & 5.4 \\
\hline $\mathrm{ABCC} 3$ & ATP-binding cassette $C$ (CFTR/MRP) & 1.09 & 8.0 \\
\hline MTIL & Metallothionein IL & I.I & 9.9 \\
\hline EGFR & Epidermal growth factor receptor & 0.95 & 8.9 \\
\hline \multicolumn{4}{|c|}{ Over-expressed in Patient I } \\
\hline pl6^ink4a & pl6^ink4a & 1.92 & 0.18 \\
\hline $\mathrm{pRb}$ & $\mathrm{pRb}$ & 1.8 & 0.21 \\
\hline \multicolumn{4}{|c|}{ Under-expressed in both patients } \\
\hline DCC & Deleted in colorectal cancer & 0.2 & 0.1 \\
\hline CASP7 & Caspase 7, apoptosis-related cysteine protease & 0.12 & 0.12 \\
\hline Bcl2-like & $2(\mathrm{Bcl}-\mathrm{w})$ & 0.15 & 0.14 \\
\hline DAP3 & Death-associated protein 3 & 0.16 & 0.18 \\
\hline \multicolumn{4}{|c|}{ Over-expressed in both patients } \\
\hline EGRI & Early growth response I & 3.1 & 3.3 \\
\hline EYA2 & Eyes absent (Drosophila) homolog 2 & 4.1 & 3.6 \\
\hline JUNB & Jun B proto-oncogene & 2.9 & 3.2 \\
\hline ZNF258 & Zinc finger protein 258 & 5.0 & 6.1 \\
\hline RGS7 & Regulator of G-protein signaling 7 & 15 & 17.2 \\
\hline EHD3 & EH domain containing 3 & 12.1 & 13.3 \\
\hline CS-I & Calcineurin-binding protein calsarcin-I & 9.8 & 10.2 \\
\hline ITPKI & Inositol I,3,4-triphosphate 5/6 kinase & 6.1 & 5.8 \\
\hline GRB2 & Growth factor receptor-bound protein 2 & 3.1 & 3.4 \\
\hline STK2 & Serine/threonine kinase 2 & 2.9 & 2.6 \\
\hline
\end{tabular}

*I/C represents the normalized ratio between test channel and control channel. 
Interestingly, p16 and pRb were expressed in Patient 1 and down-regulated in Patient 2, while EGFR was overexpressed only in Patient 2.

\section{QRT-PCR}

Statistically significant changes in gene expression at the $P=0.025$ level were seen with all transcripts analyzed. The results of real-time analysis are reported in Figure 3b.

\section{Methylation analysis of MGMT, p16, DCC, DAPK1}

DCC was found methylated in both tumors, MGMT methylated only in Patient 1, in line with the chemotherapy response, and p16 methylated only in Patient 2 since this gene is under-expressed even if not mutated (Figure 4a). Primer sequences used in methylation-specific PCR (MSP-PCR) are reported in Figure 4b.

\section{Discussion}

Unlike other cerebral gliomas, gliomatosis cerebri is a particularly invasive glioma subform, characterized by extraordinary brain spreading [2]. Like TP53 mutations, molecular alterations in gliomatosis cerebri resemble those detected in diffuse astrocytic gliomas [3]. Herrlinger et al.
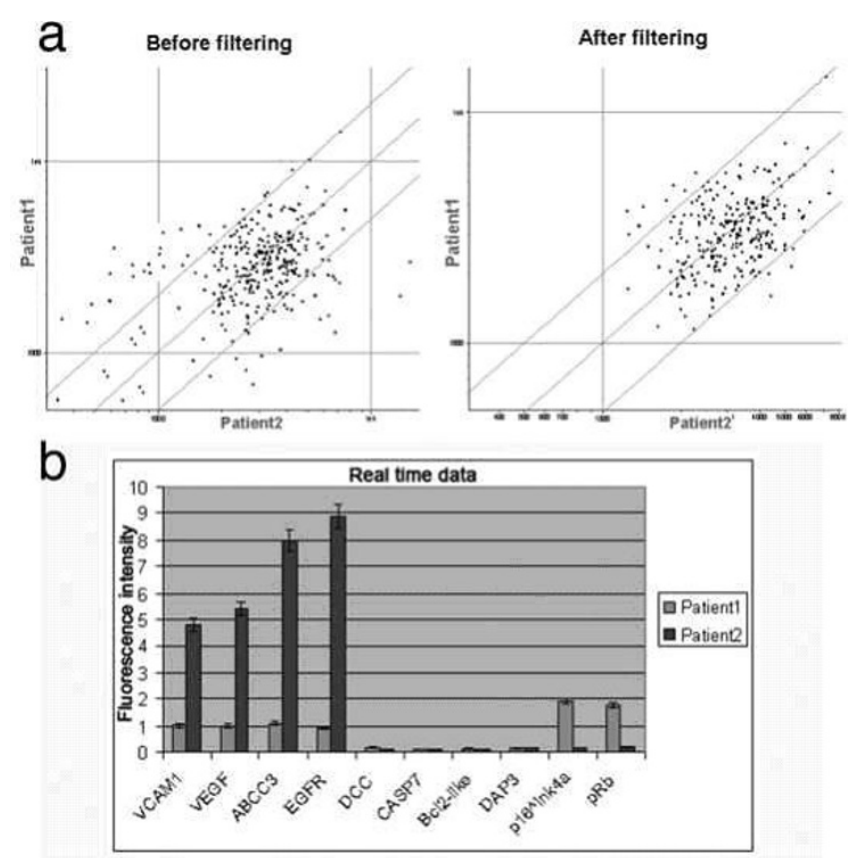

Figure 3. (a) Scatterplot showing the gene expression difference between the two patients. Threshold lines are set to a 2-fold change. The left panel shows the data before background filtering, while the right panel shows the data after filtering. (b) Histogram showing expression data regarding the selected genes by real-time RT-PCR. Normalization was conducted on beta-actin. described alterations not only in the TP53 gene, but also in PTEN [2]. Our data seem to indicate a mutually exclusive occurrence of both TP53 and PTEN mutations; in fact, Patient 1 had a mutation of TP53 without any PTEN alterations and Patient 2 exhibited a PTEN mutation with an intact TP53. Genetic aberrations lack cell cycle regulatory genes (CDKN2A, CDK4) and the strong expression of p16INK4a and pRb suggests that the pRb-dependent cell cycle checkpoint was intact in Patient 1. Patient 2 showed no genomic aberrations on the CDKN2A locus, but had a reduced p16 expression; thus, to understand the mechanism underlying its reduced expression, we investigated the status
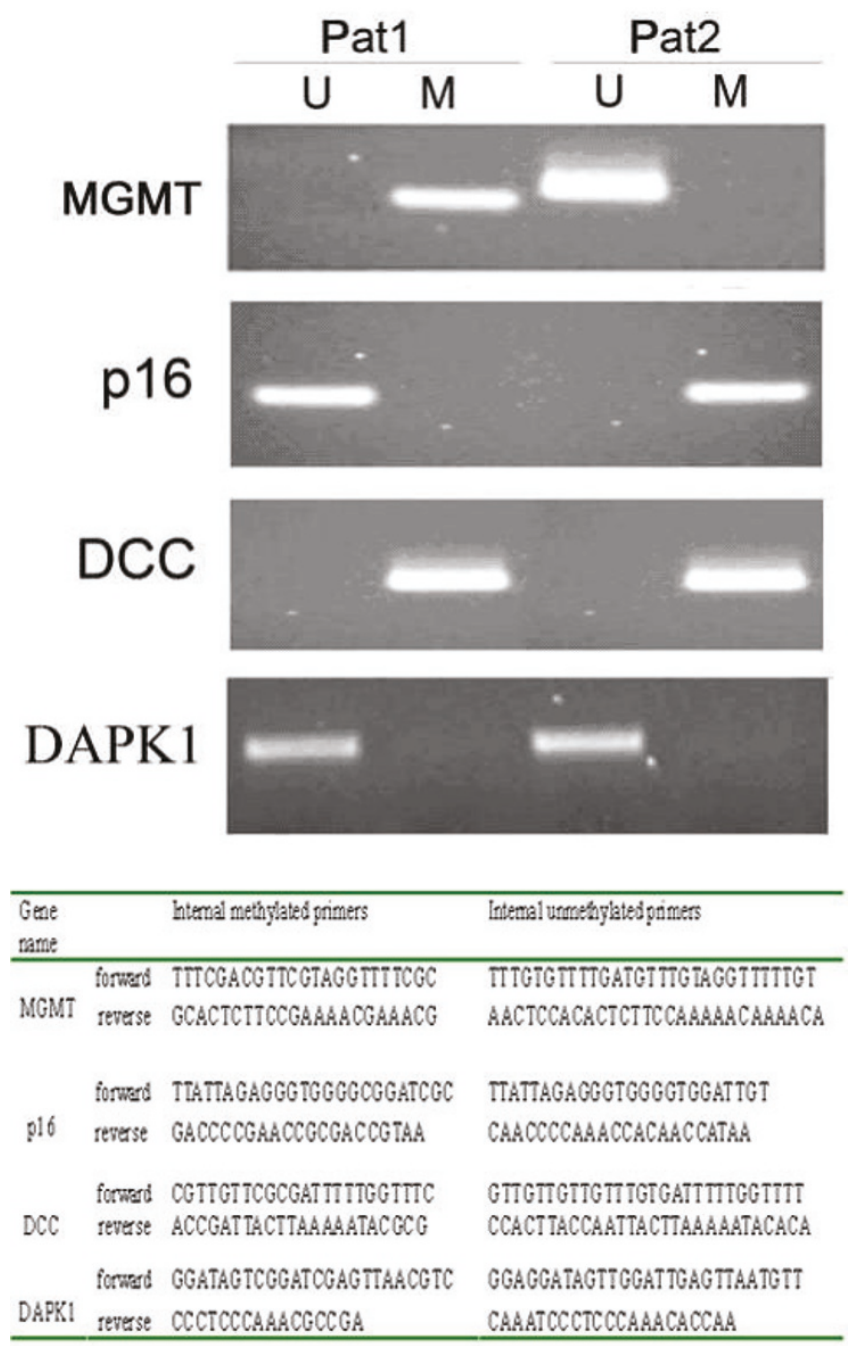

Figure 4. (a) Agarose gel electrophoresis relative to MSP products. Lines marked "U" contain products derived from unmethylated DNA templates, whereas products amplified from methylated templates are found in lines " $M$ ".

(b) Primer sequences used in the methylation-specific PCR to study the methylation status of the promoter of the following genes: MGMT, pI6, DCC and DAPK. 
of its promoter methylation and found it to be hypermethylated: the hypermethylated p16 promoter inhibited its expression [4-7]. In Patient 2, an increase was found in the EGFR copy number with its strong expression and PTEN mutation; these are all aberrations found in de novo glioblastoma. Interestingly, we found a deletion of the p27kip1 gene locus in both patients, underlining the loss of cell-cycle control. No aberrations were found in CDKN1A (p21). We found altered expressions of several genes involved in different cellular process such as those involved in the transcriptional regulation of growth control, and those involved in signal transduction cascades and cytoskeleton related genes. Two angiogenesis related genes, wellknown in glioma biology, VCAM1 and VEGF, were overexpressed in Patient 2 reflecting the high degree of neovascularization. Genes involved in drug resistance, ABCC3 and metallothioneins, which could play a role in drug resistance, were highly expressed in Patient 2's tumor tissue, explaining his non-responsiveness to chemotherapy. One hallmark of tumors is the lack of control of apoptosis linked genes (CASP7, BCLW, DAP3), found to be downregulated in both tumors. The absence of DCC function, associated with several types of tumors, appeared to also affect our patients. Moreover, we investigated the methylation status of the DNA repair protein gene O6-methylguanine-methyltransferase (MGMT), which is a marker of resistance to chemotherapeutic alkylating agents, including temozolomide (TMZ), in high-grade gliomas, particularly glioblastomas [8]. Since the protein is able to repair DNA lesions induced by alkylating factors, methylation of MGMT promoter (M-MGMT) results in better responsiveness to treatment with M-MGMT favoring the alkylating agents function; in fact, Everhard et al. found that M-MGMTP patients had a significantly longer progression-free survival after TMZ than U-MGMTP patients [9]. Our data confirm such a correlation, in that Patient 1 with M-MGMT responded to $\mathrm{TMZ}$, while no response was observed in Patient 2 with U-MGMT.

\section{Conclusions}

We report two cases of GC Type II carrying similar clinical and histological patterns but with distinct molecular and prognostic features. The poor prognosis of Patient 2 was underlined by molecular analysis which showed overexpression of the angiogenesis related genes VCAM1 and $V E G F$, reflecting the high degree of neovascularization present. Moreover, in the same patient, we found a high expression of genes involved in drug resistance such as ABCC3 (multidrug resistance protein 3, MRP3) and metallothioneins, which could play a role in intrinsic drug resistance, and, along with unmethylated MGMT status, may explain the lack of response to chemotherapy and overall poor prognosis of this patient. Current prognostic stratification of GC Type II is mainly based on tumor grade as assessed by the pathologist; our data, according to a few literature reports, seem to suggest that molecular characterization is required in order to provide proper subtyping and to identify targeted treatment strategies.

\section{Consent}

Written informed consent was obtained from both patients for publication of this case report and any accompanying images. A copy of the written consent is available for review by the Editor-in-Chief of this journal.

\section{Competing interest}

The authors declare that they have no competing interests.

\section{Authors' contributions}

PID'U: study concept and design, patient care, acquisition of data, literature review. OFD'U: study concept and design, data analysis, interpretation of data. SM: study concept and design, data analysis, literature review. CS: drafting and revising the manuscript. AD: interpretation of data, drafting the manuscript. FS: acquisition of data, drafting the manuscript. AC: acquisition of data, drafting the manuscript. GL: study concept and design, revising the manuscript. CDG: patient care, data analysis, literature review. AM: patient care, revising the manuscript. PC: patient care, literature review, drafting and revising the manuscript.

\section{Acknowledgment}

Thanks to Cora Stern for the English revision.

\section{References}

I. Fuller GN, Kros JM: Gliomatosis cerebri. In WHO Classification of Tumours of the Central Nervous System. Edited by Louis DN, Ohgaki H, Wiestler OD, Cavenee WK. Lyon, IARC; 2007:50-52.

2. Herrlinger U, Felsberg J, Küker W, Bornemann A, Plasswilm L, Knobbe CB, Strik H, Wick W, Meyermann R, Dichgans J, Bamberg M, Reifenberg G, Weller M: Gliomatosis cerebri: molecular pathology and clinical course. Ann Neurol 2002, 52:390-399.

3. Cavenee WK: High-grade gliomas with chromosome Ip loss. J Neurosurg 2000, 92:1080-1081.

4. Watanabe K, Peraud A, Gratas C, Wakai S, Kleihues P, Ohgaki H: p53 and PTEN gene mutations in gemistocytic astrocytomas. Acta Neuropathol 1998, 95:559-564.

5. Tohma Y, Gratas C, Biernat W, Peraud A, Fukuda M, Yonekawa Y, Kleihues P, Ohgaki H: PTEN (MMACI) mutations are frequent in primary glioblastomas (de novo) but not in secondary glioblastomas. J Neuropathol Exp Neurol 1998, 57:684-689.

6. Koopmann J, Maintz D, Schild S, Schramm J, Louis DN, Wiestler OD, von Deimling A: Multiple polymorphisms, but no mutations, in the WAFI/CIPI gene in human brain tumours. $\mathrm{Br} J$ Cancer 1995, 72:1230-1233.

7. Naumann U, Weit S, Rieger L, Meyermann R, Weller M: p27 modulates cell cycle progression and chemosensitivity in human malignant glioma. Biochem Biophys Res Commun 1999, 26 I:890-896.

8. Hegi ME, Diserens AC, Gorlia T, Hamou MF, de Tribolet N, Weller M, Kros JM, Hainfellner JA, Mason W, Mariani L, Bromberg JE, Hau P, Mirimanoff RO, Cairncross JG, Panzer RC, Stupp R: MGMT gene silencing and benefit from temozolomide in glioblastoma. N Engl J Med 2005, 352:997-1003.

9. Everhard S, Kaloshi G, Crinière E, Benouaich-Amiel A, Lejeune J, Marie Y, Sanson M, Kujas M, Mokhtari K, Hoang-Xuan K, Delattre JY, Thillet J: MGMT methylation: A marker of response to temozolomide in low-grade gliomas. Annals of Neurology 2006, 60:740-743. 